\title{
A New Robust Color Image Watermarking Scheme Based on 3D-DCT
}

\author{
Xiangguang Xiong \\ School of Mathematics and Computer Science, Guizhou Normal University, Guiyang, China \\ Email: xxg0851@163.com
}

Received 7 September 2015; accepted 16 October 2015; published 23 October 2015

\begin{abstract}
In this paper, for color image copyright protection application, a new and blind color image watermarking algorithm based on quantitation method in three dimensional discrete cosine transform (3D-DCT) is proposed. Firstly, the original RGB color image is divided into non-overlapping blocks sized $8 \times 8 \times 3$, and then performs 3D-DCT transform on each block. Secondly, embed a bit watermarking signal into each block's 3D-DCT direct-current (DC) coefficient by quantization method. The results show that the proposed scheme has very good imperceptibility and robustness against the common image processing attacks. Compared with similar algorithm, the proposed scheme has better robustness performance for most attacks.
\end{abstract}

\section{Keywords}

Color Image Watermarking, Three Dimensional Discrete Cosine Transform (3D-DCT), Quantitative Method, Robustness

\section{Introduction}

With the development of multimedia technology, computer technology and network technology, the digital multimedia can be completely reconstructed without any distortion. However, many new issues have emerged, such as the security of computer network transmission and the copyright protection of digital products [1]. For copyright protection application of digital multimedia, copyright owner generally embeds a watermarking signal (may be a number, a text or an image) into original digital multimedia to verify its copyright.

Current, most watermarking algorithms usually use gray-scale image as original cover signal. If the original cover image is color image, it can be as a three-dimensional signal and can be performed 3D transform firstly, and then embed watermarking signal into selected frequency domain coefficients [2] [3]. However, some researchers proposed color image digital watermarking algorithms embed directly watermarking signal into each channel or selected channel [1]. Besides, some researchers first convert color space from RGB to YCbCr or YUV and then select Y channel for watermarking embedding [4]-[10]. According to the watermarking embedding domain, digital watermarking algorithms can be classified into two categories: spatial domain algorithm and transform domain algorithm. Generally, spatial domain method has worse robustness performance than transform domain method. Hence, most watermarking algorithms usually embed watermarking signal into transform domain coefficients. For transform domain algorithms, first the cover image or image block is per- 
formed by DCT [4]-[10]. And then embed the watermarking signal into corresponding transform domain coefficients by proposed embedding rule.

In this paper, a new and blind robust RGB color image watermarking scheme based on 3D-DCT transform is proposed. Firstly, the original RGB color image is divided into non-overlapping blocks sized $8 \times 8 \times 3$, and then performs 3D-DCT transform on each block. Secondly, embed a bit watermarking signal into each block's 3D-DCT direct-current (DC) coefficient by quantization method. The proposed scheme can not only achieve good imperceptibility but also against the common image processing attacks, such as adding noise, filtering, cropping, JPEG compression, scaling and rotating. Compared with similar watermarking algorithm, it has a better robustness performance for most attacks.

The rest of the paper is organized as follows. Section 2 briefly defines 3D-DCT transform and its inverse transform. Section 3 proposes detailed watermarking embedding and extraction procedures. The corresponding experimental results are given in Section 4. Finally, the conclusions are drawn in Section 5.

\section{3D-DCT Transform}

For a given 3D volume data sized $n_{x}, n_{y}, n_{z}$, the 3D-DCT transform is defined as follows.

$$
F(a, b, c)=C(a) C(b) C(c)\left[\sum_{x=0}^{n_{x}-1} \sum_{y=0}^{n_{y}-1} \sum_{z=0}^{n_{z}-1} f(x, y, z) \frac{(2 x+1) a \pi}{2 n_{x}} \frac{(2 y+1) b \pi}{2 n_{y}} \frac{(2 z+1) c \pi}{2 n_{z}}\right]
$$

where $C(a)=\left\{\begin{array}{l}\sqrt{1 / n_{x}}, a=0 \\ \sqrt{2 / n_{x}}, a \neq 0\end{array}, C(b)=\left\{\begin{array}{l}\sqrt{1 / n_{y}}, b=0 \\ \sqrt{2 / n_{y}}, b \neq 0\end{array}, C(c)=\left\{\begin{array}{l}\sqrt{1 / n_{z}}, c=0 \\ \sqrt{2 / n_{z}}, c \neq 0\end{array}, \quad f(x, y, z)\right.\right.\right.$ is the pixel value at position $(x, y, z)$, and $F(a, b, c)$ is 3D-DCT transform coefficient. The inverse 3D-DCT transform is defined as below.

$$
f(x, y, z)=\sum_{a=0}^{n_{x}-1} \sum_{b=0}^{n_{y}-1} \sum_{c=0}^{n_{z}-1}\left[C(a) C(b) C(c) F(a, b, c) \frac{(2 x+1) a \pi}{2 n_{x}} \frac{(2 y+1) b \pi}{2 n_{y}} \frac{(2 z+1) c \pi}{2 n_{z}}\right]
$$

\section{Proposed Scheme}

For the proposed scheme, it consists of two phases: watermarking embedding procedure and extraction procedure.

\subsection{Watermarking Embedding Procedure}

The proposed watermarking embedding procedure is showed in Figure 1. The detailed steps are described as follows.

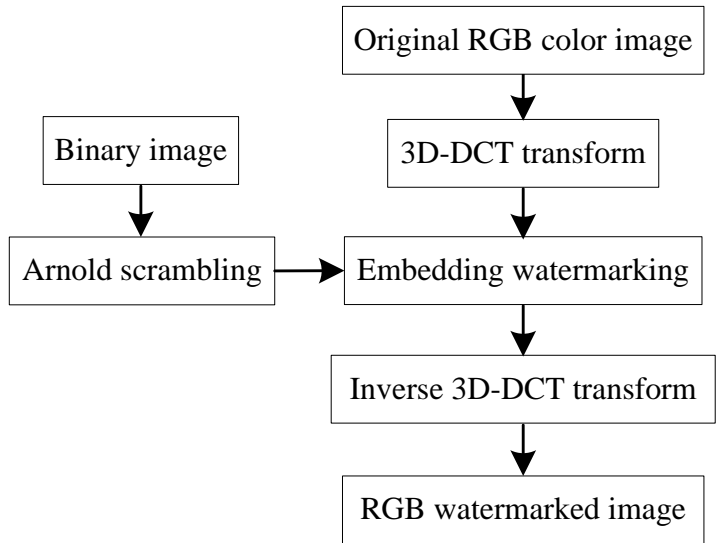

Figure 1. Flow diagram of watermarking embedding procedure. 
Step 1. According to selected iteration times $n$, encrypt binary watermarking image with Arnold transform to obtain an encrypted watermarking image.

Step 2. For a given color image, it is divided into blocks sized $8 \times 8 \times 3$.

Step 3. For each image block, 3D-DCT transform is performed on it.

Step 4. Suppose the watermarking embedding strength is $Q$. Modify the DC coefficient to embed watermarking signal. The watermarking embedding rule is defined as bellow.

$$
D C^{\prime}=\left\{\begin{array}{l}
D C+Q / 2-\bmod (D C+0.75 Q, Q), \text { if } w=1 \\
D C+Q / 2-\bmod (D C-0.75 Q, Q), \text { if } w=0
\end{array}\right.
$$

Step 5. Perform inverse 3D-DCT transform on new DC coefficient and other coefficients to obtain an embedded image block. When all blocks are embedded, the final watermarked image is obtained.

\subsection{Watermarking Extraction Procedure}

The watermarking extraction procedure is just the inverse procedure of the watermarking embedding procedure. The extraction procedure of the watermarking is blind, which does not need the original cover image. The watermarking extraction procedure is showed in Figure 2. The watermarking extraction steps can be described as below.

Step 1. For a given watermarked color image, it is divided into blocks sized $8 \times 8 \times 3$.

Step 2. For each image block, 3D-DCT transform is performed on it.

Step 3. The binary watermarking bits can be extracted as follows.

$$
w^{\prime}=\left\{\begin{array}{l}
1, \text { if } \bmod (D C, Q) \geq Q / 2 \\
0, \text { else }
\end{array}\right.
$$

Step 4. According to selected iteration times $n$ during watermarking embedding procedure, decrypt the extracted binary watermarking signal using inverse Arnold transform to obtain final extracted watermarking image.

\section{Experimental Results}

To test the robustness of proposed scheme, the original watermarking signal is a binary image sized $64 \times 64$ and is showed in Figure 3. The original cover images are four RGB true color image sized $512 \times 512 \times 3$, namely Airplane, Sailboat, Peppers and Lena, as shown in Figure 4. The threshold $Q$ of the proposed scheme defined in Section III is 60 .

\subsection{Imperceptibility Test}

The Peak Signal to Noise Ratio (PSNR) is used to evaluate the distortion between the cover image $x$ and the watermarked image $x^{\prime}$, which is defined as below.

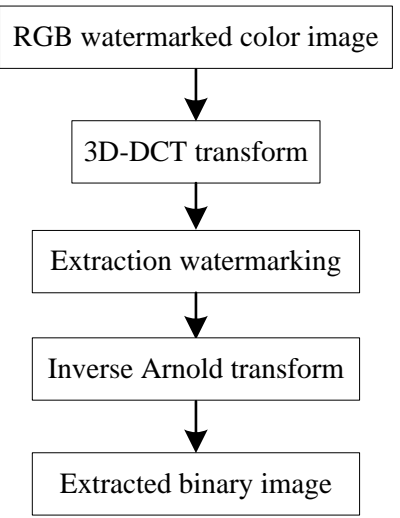

Figure 2. Flow diagram of watermarking extraction procedure. 


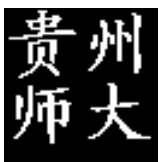

Figure 3. Original binary watermarking image.

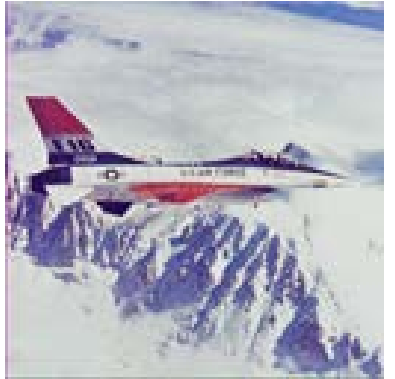

(a) Original Airplane

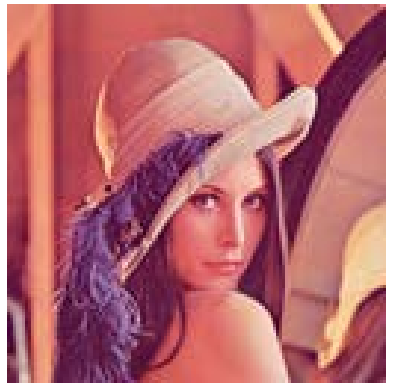

(e) Original Lena

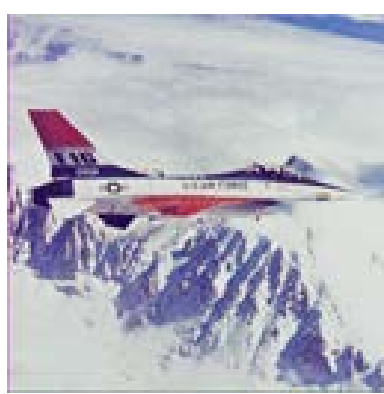

(b) Watermarked Airplane

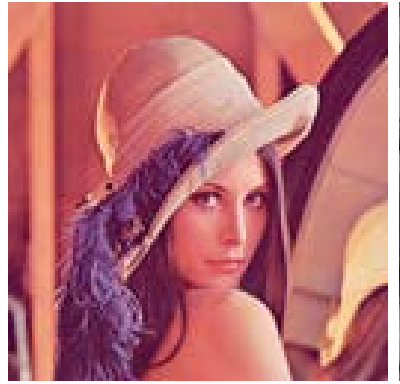

(f) Watermarked Lena

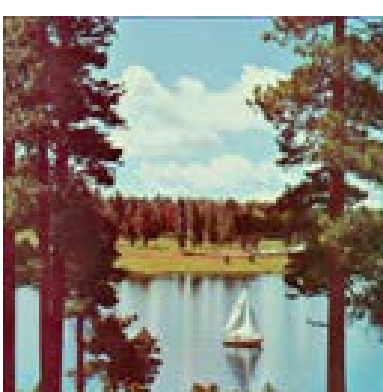

(c) Original Sailboat

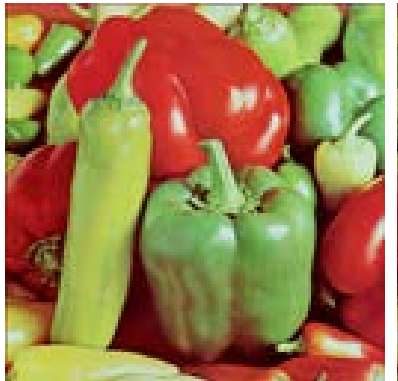

(g) Original Peppers

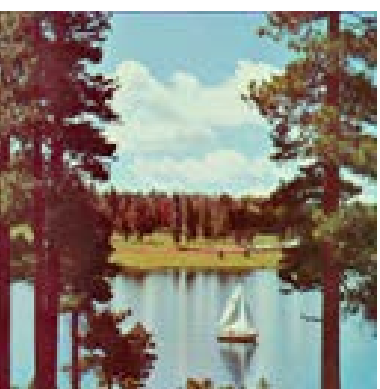

(d) Watermarked Sailboat

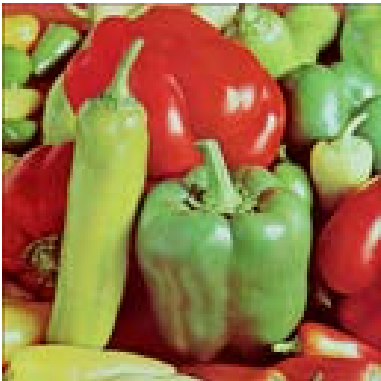

(h) Watermarked Peppers

Figure 4. Original images and watermarked images.

$$
P S N R=10 \times \log _{10}\left(\frac{255^{2}}{M S E}\right)
$$

The mean square error (MSE) between $x$ and $x^{\prime}$ is defined as blew.

$$
M S E=\frac{1}{M \times M} \times \sum_{i=1}^{M} \sum_{j=1}^{M}\left(x_{i, j}-x_{i, j}^{\prime}\right)^{2}
$$

where $M$ is width of original cover image.

The average PSNR between the original RGB image and its watermarked image are shown in Table 1. From Table 1, it can be seen that the proposed scheme and the algorithm in [3] have good visual quality of watermarked image. The average PSNR for proposed scheme are about $45.85 \mathrm{~dB}$.

\subsection{Robustness Test}

The Normalized Cross-Correlation $(N C)$ is used to measure the similarity between the original binary watermarking signal $w$ and the extracted binary watermarking signal $w^{\prime}$, which is defined as follows.

$$
N C=\frac{1}{N \times N} \times \sum_{i=1}^{N} \sum_{j=1}^{N}\left(w_{i, j} \oplus w_{i, j}^{\prime}\right)
$$

where $N$ is width of binary watermarking signal, $\oplus$ symbol represents XOR operation.

To verify the robustness of the proposed scheme, the watermarked image is first attacked by common image processing. And then the embedded watermarking signal is extracted from attacked watermarked image. The results for watermarked Lena color image are shown in Figure 5 (The results of other images are similar with Lena image). It can be easily seen that the extracted watermarking images can be discriminated by human visual 
system. These results show that the proposed scheme can achieve excellent robustness against common image processing attacks.

\subsection{Performance Comparison}

To test the superiority of the proposed scheme, we compared the proposed scheme with similar algorithm based on 3D-DCT in [3]. Under the same conditions, the results between PSNR and NC are shown in Table 2. From Table 2, it can be easily seen that the proposed scheme and the algorithm in [3] have almost same PSNR values after various attack, but the proposed scheme has better robustness performance.

Figure 6 gives the results between the proposed scheme and the algorithm in [3] on Lena image. From Figure 6, it can be easily seen that compared with the algorithm in [3], the proposed scheme has better robustness against most common image processing attacks.

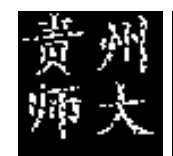

(a)

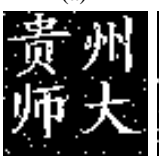

(e)

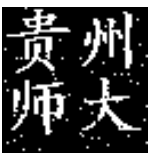

(b)

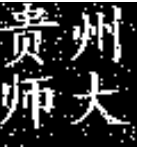

(f)

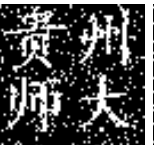

(c)

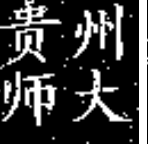

(g)

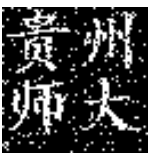

(d)

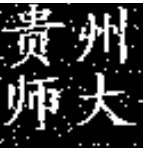

(h)

Figure 5. Extracted watermarking image. Various attacks have been utilized independently to each component each time for Lena image. (a) Crop (1/4); (b) JPEG compression (Q = 80); (c) median filter (3 × 3); (d) rotate (10 $0^{\circ}$; (e) scale (0.5); (f) salt $\&$ peppers noise (0.001); (g) speckle noise $(0.001)$; (h) wiener filter $(3 \times 3)$.

Table 1. PSNR comparisons between the proposed scheme and the algorithm in [3].

\begin{tabular}{ccc}
\hline Test image & Proposed scheme & {$[3]$} \\
\hline Airplane & 45.8905 & 45.4424 \\
Lena & 45.8888 & 45.4374 \\
Peppers & 45.9074 & 45.5219 \\
Sailboat & 45.7450 & 45.4507 \\
\hline
\end{tabular}

Table 2. PSNR and NC comparisons between the proposed scheme and the algorithm in [3].

\begin{tabular}{|c|c|c|c|c|c|}
\hline \multirow{2}{*}{ Attack type } & \multirow{2}{*}{ Intensity } & \multicolumn{2}{|c|}{ Proposed scheme } & \multicolumn{2}{|c|}{ [3] } \\
\hline & & PSNR & $N C$ & PSNR & $N C$ \\
\hline \multirow{3}{*}{ Compression } & JPEG compression $(\mathrm{Q}=60)$ & 32.4461 & 0.8564 & 32.4273 & 0.9004 \\
\hline & JPEG compression $(\mathrm{Q}=70)$ & 32.9402 & 0.9163 & 32.9126 & 0.9126 \\
\hline & JPEG compression $(\mathrm{Q}=80)$ & 33.6178 & 0.9844 & 33.5874 & 0.9102 \\
\hline \multirow{2}{*}{ Noise } & Speckle noise & 35.2496 & 0.9878 & 35.1973 & 0.9138 \\
\hline & Salt \& pepper noise & 35.0859 & 0.9709 & 34.7111 & 0.9817 \\
\hline \multirow{4}{*}{ Filtering } & Median filtering $(3 \times 3)$ & 33.9339 & 0.9194 & 33.9918 & 0.9133 \\
\hline & Median filtering $(5 \times 5)$ & 30.6079 & 0.8027 & 30.6583 & 0.7869 \\
\hline & Wiener filtering $(3 \times 3)$ & 35.7532 & 0.9732 & 35.8472 & 0.9282 \\
\hline & Wiener filtering $(5 \times 5)$ & 33.5019 & 0.7832 & 33.5941 & 0.7798 \\
\hline \multirow{2}{*}{ Rotating } & 5 degree & 20.0438 & 0.9407 & 20.0449 & 0.8953 \\
\hline & 10 degree & 17.5140 & 0.9390 & 17.5147 & 0.8857 \\
\hline \multirow{2}{*}{ Rescaling } & Scale (0.5) & 33.1163 & 0.9846 & 33.1603 & 0.9404 \\
\hline & Scale (2) & 41.1787 & 1.0000 & 41.1122 & 0.9412 \\
\hline \multirow{2}{*}{ Cropping } & Cropping (1/16) & 18.4751 & 0.9895 & 18.4741 & 0.9661 \\
\hline & Cropping (1/4) & 12.1114 & 0.9563 & 12.1112 & 0.8599 \\
\hline
\end{tabular}



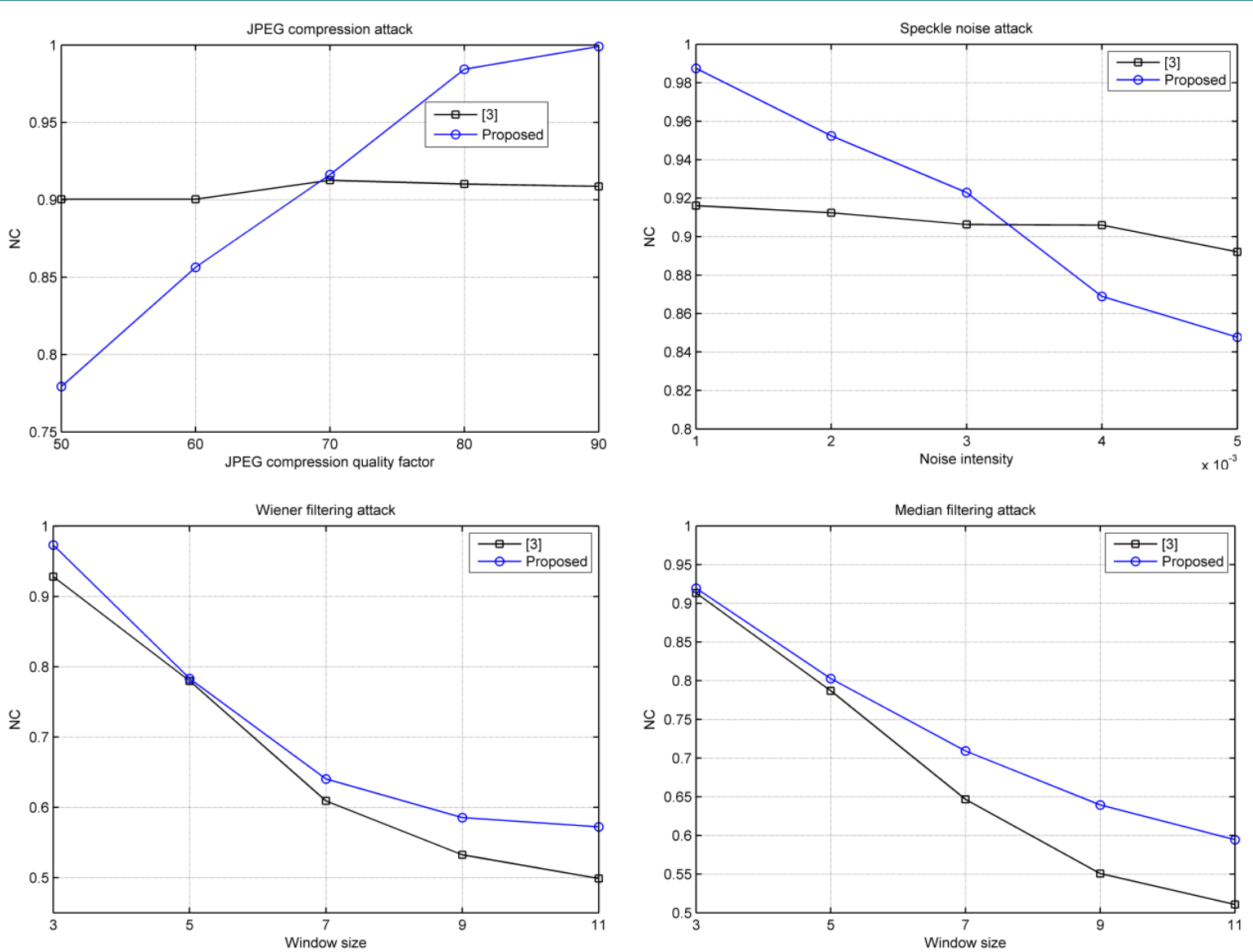

Figure 6. Performance comparison between the proposed scheme and the algorithm in [3].

\section{Conclusion}

In this paper, a new and blind color image watermarking scheme based on quantization modulation in 3D-DCT domain is proposed. The proposed scheme embeds only a bit into each block's 3D-DCT DC coefficient by quantization rule. Experimental results show that the proposed scheme has good imperceptibility and robustness against common image processing attacks. The comparison results between the proposed scheme and the algorithm in [3] show that the proposed scheme has better performance for most attacks.

\section{Acknowledgements}

This work was funded by the Joint Foundation of Department of Science and Technology of Guizhou Province and Guizhou Normal University (Qian-Ke-He LH Zi [2014] 7041).

\section{References}

[1] Barni, M., Bartolini, F. and Piva, A. (2002) Multichannel Watermarking of Color Images. IEEE Transactions on Circuits and Systems for Video Technology, 12, 142-156. http://dx.doi.org/10.1109/76.993436

[2] Fu, Y. and Wang, H. (2009) Secure Spread Image Watermarking Scheme in 3D-DCT Domain. Proceedings of 2nd International Congress on Image and Signal Processing, 1-4. http://dx.doi.org/10.1109/cisp.2009.5303499

[3] Fu, Y. (2009) Robust Image Watermarking Scheme Based on 3D-DCT. Proceedings of Sixth International Conference on Fuzzy Systems and Knowledge Discovery, 5, 437-441. http://dx.doi.org/10.1109/fskd.2009.19

[4] Feng, S., Lin, D., Shie, S.C. and Guo, J.Y. (2010) Improving the Robustness of DCT Based Image Watermarking against JPEG Compression. Computer Standards \& Interface, 32, 54-60. http://dx.doi.org/10.1016/j.csi.2009.06.004

[5] Xie, B. (2011) A Blind Watermarking Algorithm Based on Color Space Conversion in DCT Domain. Proceedings of 2011 International Conference on Control, Automation and Systems Engineering, 1-3. 
http://dx.doi.org/10.1109/iccase.2011.5997612

[6] Zhong, Q.C. and Zhu, Q.X. (2009) A DCT Domain Color Watermarking Scheme Based on Chaos and Multilayer Arnold Transformation. Proceedings of International Conference on Networking and Digital Society, 209-212.

[7] Zhang, T. and Du, Y. (2009) A Digital Watermarking Algorithm for Color Images Based on DCT. Proceedings of International Conference on Information Engineering and Computer Science, 1-4. http://dx.doi.org/10.1109/iciecs.2009.5364628

[8] Ahmidi, N. and Safabakhsh, R. (2004) A Novel DCT-Based Approach for Secure Color Image Watermarking. Proceedings of International Conference on Information Technology: Coding and Computing, 2, 709-713. http://dx.doi.org/10.1109/itcc.2004.1286738

[9] Zhou, Y. and Liu, J. (2009) Blind Watermarking Algorithm Based on DCT for Color Images. Proceedings of 2nd International Congress on Image and Signal Processing, 1-3. http://dx.doi.org/10.1109/cisp.2009.5303681

[10] Al-Gindy, A., Al-Ahmad, A., Qahwaji, R. and Tawfik, A. (2009) Watermarking of Color Images in the DCT Domain Using Y Channel. Proceedings of IEEE/ACS International Conference on Computer Systems and Applications, 10251028. 